\title{
HGF stimulates proliferation through the HGF/c-Met pathway in nasopharyngeal carcinoma cells
}

\author{
RUI SUN ${ }^{1,2^{*}}$, QING ZHANG $^{3 *}$, LING GUO $^{1,2}$, MING-YUAN CHEN $^{1,2}$, YING SUN $^{1,2}$, BRIAN CAO $^{4}$ and JIAN SUN ${ }^{1,2}$ \\ ${ }^{1}$ State Key Laboratory of Oncology in Southern China; ${ }^{2}$ Cancer Center, Sun Yat-sen University, Guangzhou 510060; \\ ${ }^{3}$ Key Laboratory of Gene Engineering of the Ministry of Education, School of Life Sciences, Sun Yat-sen University, \\ Guangzhou 510275, P.R. China; ${ }^{4}$ Van Andel Research Institute, Grand Rapids, MI 49503, USA
}

Received December 6, 2011; Accepted January 16, 2012

DOI: $10.3892 / 01.2012 .613$

\begin{abstract}
Hepatocyte growth factor (HGF) and its receptor c-Met are important in the development and homeostasis of a variety of human malignancies. However, the role of the $\mathrm{HGF} / \mathrm{c}-$ Met signaling pathway in nasopharyngeal carcinoma (NPC) has not been clearly elucidated. This study examined the effect of $\mathrm{HGF} / \mathrm{c}-\mathrm{Met}$ on proliferation and migration in several NPC cell lines. RT-PCR was used to detect the HGF gene in CNE-1, CNE-2, HK-1, HONE-1 and SUNE-1 NPC cells. However, HGF gene expression was not detected in any of these cells. Using immunoblotting analysis, the Met25 protein was identified in HONE-1, HK-1 and CNE-1 cells. Results from fluorescence-activated cell sorting (FACS) analysis revealed that anti-Met $25 \mathrm{mAb}$ specifically bound Met-expressing HONE-1, HK-1 and CNE-1 cells. It was further demonstrated that exogenous HGF was able to stimulate the proliferation of HONE-1 and HK-1 cells and the healing of scrape wounds in HONE-1 NPC cells. Our results reveal the potential therapeutic applications of combination therapy with antibodies targeting HGF in NPC patients.
\end{abstract}

Correspondence to: Professor Brian Cao, Van Andel Research Institute, 333 Bostwick Avenue NE, Grand Rapids, MI 49503, USA E-mail: brian_cao@yahoo.cn

Dr Jian Sun, Cancer Center, Sun Yat-sen University, 651 Dongfeng East Road, Guangzhou 510060, P.R. China

E-mail: sunjian@sysucc.org.cn

*Contributed equally

Abbreviations: HGF, hepatocyte growth factor; NPC, nasopharyngeal carcinoma; FACS, fluorescence-activated cell sorting; PBS, phosphate-buffered saline; MTT, 3-(4,5-dimethylthiazol-2-yl)-2,5diphenyltetrazolium bromide; GAPDH, glyceraldehyde-3-phosphate dehydrogenase

Key words: nasopharyngeal carcinoma, HGF/c-Met, proliferation, migration

\section{Introduction}

Nasopharyngeal carcinoma (NPC) is one of the most common types of cancer in South East Asia, and remains a cause of high morbidity in Southern China (1). In addition to its ability for rapid growth, NPC has a tendency to invade the adjacent regions and metastasize to the regional lymph nodes and distant organs. Despite the sequela of radiation, radiotherapy remains the standard treatment for NPC $(2,3)$. However, only early stage NPC cases are able to obtain effective results, including good prognosis and function. The combination of radiotherapy and chemotherapy is crucial in the therapy of locoregional advanced cases of NPC (4). Although over $95 \%$ of biopsies belong to the WHO type II or III classification, which are sensitive to radiotherapy and chemotherapy, there are many treatment-failure cases (5). Thus, there is a requirement for new therapeutic targets and a better understanding of the mechanisms involved in the metastasis of NPC.

The Met receptor tyrosine kinase and its ligand, hepatocyte growth factor (HGF), are overexpressed and/or activated in a variety of human malignancies. The hepatocyte growth factor/ scatter factor $(\mathrm{HGF} / \mathrm{SF})$ and its receptor Met are important in the development, homeostasis, tumorigenesis, angiogenesis, invasion and metastasis of human malignancies (6). However, the mechanisms of the HGF/c-Met signaling pathway that contribute to the invasiveness of malignancies remain unknown. The Met receptor is frequently overexpressed in NPC patients, and high expression is associated with short patient survival (7). The availability of the Met receptor has been shown to modify NPC cell response to HGF. This finding enhanced our understanding of the mechanisms of signaling transduction in the HGF-induced progression of NPC. However, the role of the HGF/c-Met signaling pathway in NPC has not been clearly elucidated. In this study, we detected the effect of HGF/c-Met on proliferation and migration in several NPC cell lines. Our results suggest the potential administration in therapeutic combination with antibodies to c-Met in NPC patients.

\section{Materials and methods}

Cell lines and reagents. Five human NPC cell lines (two welldifferentiated NPC cell lines, CNE-1 and HK-1, and three 
poorly differentiated NPC cell lines, CNE-2, HONE-1, and SUNE-1) were maintained in RPMI-1640 (Invitrogen Life Technologies, Carlsbad, CA, USA), and were supplemented with $10 \%$ fetal bovine serum (FBS, Gibco, Carlsbad, CA, USA), $1 \mathrm{U} / \mathrm{ml}$ penicillin $\mathrm{G}$, and $1 \mathrm{mg} / \mathrm{ml}$ streptomycin at $37^{\circ} \mathrm{C}$ and $5 \% \mathrm{CO}_{2}$. The $\mathrm{S} 114$ cells (NIH $3 \mathrm{~T} 3$ cells transformed with human HGF/SF and Met) (8) and the SK-LMS-1 human leiomyosarcoma cell line (9) were maintained in DMEM (Gibco) containing $10 \% \mathrm{FBS}$ at $37^{\circ} \mathrm{C}$ and $5 \% \mathrm{CO}_{2}$. S-114 cells stably co-express human Met and HGF, resulting in autoactivation of the Met receptor. The SK-LMS-1 cell line was derived from a human leiomyosarcoma (smooth-muscle tumor); these cells express a high level of the Met receptor, but only a small quantity of its ligand $\mathrm{HGF} / \mathrm{SF}$, and respond mitogenically to exogenous HGF/SF. The SK-LMS-1 cell line was used as the control in the cell proliferation assay, as it is able to proliferate under exogenous HGF. The HGF/SF was prepared from S114 cells. Anti-HGF/SF neutralizing antibodies were generated as previously described (10).

$R T-P C R$. Total RNA was extracted from cell line pellets using TRIzol reagent, and reverse transcription was performed using the SuperScript II RT kit (Invitrogen, Grand Island, NY, USA; no. 10928034) with total RNA (1 $\mu \mathrm{g})$ according to the manufacturer's instructions. The HGF mRNA expression levels were detected by conventional RT-PCR with Taq DNA Polymerase, Recombinant (Invitrogen, no. 10342-020). Glyceraldehyde-3-phosphate dehydrogenase (GAPDH) was used as the internal control.

The specific primers for HGF and GAPDH were designed by Primeriers software. The PCR reaction was performed according to the manufacturer's instructions. The primers used were: HGF, forward: 5'-CTACACTGGATTGATCAACTAT-3' and reverse: 5'-AGTAGTTGTCTTAGGATTGTTG-3'; and GAPDH, forward: 5'-TTGCCATCAATGACCCCTTCA-3' and reverse: 5'-CGCCCCACTTGATTTTGGA-3'.

The PCR conditions were as follows: amplification reaction protocol was performed for 35 cycles consisting of $30 \mathrm{sec}$ at $94^{\circ} \mathrm{C}$ (denaturation), annealing $30 \mathrm{sec}$ at $45^{\circ} \mathrm{C}$ and extension $30 \mathrm{sec}$ at $72^{\circ} \mathrm{C}$. The PCR products were separated on a $2 \%$ agarose gel, stained with ethidium bromide and visualized by the Bio-Rad Imaging System (Hercules, CA, USA).

Western blot analysis. S114, NIH 3T3, CNE-1, CNE-2, HK-1, HONE-1 and SUNE-1 cells were harvested and centrifuged. The whole cell lysates were prepared by adding 2X SDS sample buffer (125 mmol/1 Tris-HCl, pH 6.8, 2\% SDS, $20 \%$ glycerol, $0.02 \mathrm{mg} / \mathrm{ml}$ bromophenol blue and $5 \%$ mercaptoethanol). Equal amounts of protein $(30 \mu \mathrm{g} / \mathrm{sample}$ as determined by UV spectrometry) were electrophoresed on $12 \%$ SDS-PAGE gels and transferred to nitrocellulose membranes. The membranes were then blocked for $60 \mathrm{~min}$ at room temperature with 5\% non-fat dry milk/TBS-Tween-20 and reacted with the appropriate antibodies for Met (1:500 dilution in blocking buffer) overnight at $4{ }^{\circ} \mathrm{C}$ with constant agitation. Following incubation with the primary antibody, membranes were washed in TBS-Tween-20 and incubated with horseradish peroxidase-conjugated secondary antibodies (1:5000 dilution in blocking buffer) for $1 \mathrm{~h}$ at room temperature. Proteins were then visualized by incubation

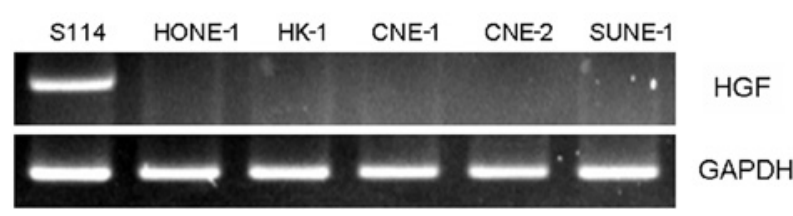

Figure 1. HGF gene expression in the CNE-1, CNE-2, HK-1, HONE-1, SUNE-1 and S114 cell lines using RT-PCR is shown. GAPDH was used as the internal control. Only the S114 cell line expressed the HGF gene. There was no HGF gene expression in any of the other five NPC cell lines. HGF, hepatocyte growth factor; NPC, nasopharyngeal carcinoma; GAPDH, glyceraldehyde-3-phosphate dehydrogenase.

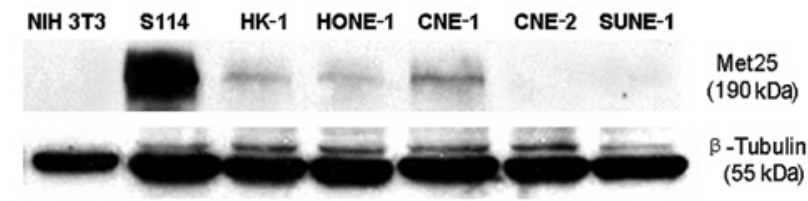

Figure 2. The Met25 protein was only expressed in the HONE-1, HK-1 and CNE-1 cell lines, but was not expressed in the CNE-2 and SUNE-1 cell lines. $\beta$-tubulin was used as the internal control.

with enhanced chemiluminescence detection reagents (Cell Signaling, No. 7072), followed by exposure to radiograph film (Kodak, No. 6535876).

Fluorescence-activated cell sorting (FACS) analysis. The whole cells, including S114, NIH 3T3, CNE-1, CNE-2, HK-1, HONE-1 and SUNE-1, were blocked using 5\% milk blocking buffer for $30 \mathrm{~min}$ at $4^{\circ} \mathrm{C}$, incubated with $2 \mathrm{mg} / \mathrm{ml}$ anti-hHGF monoclonal antibody for $60 \mathrm{~min}$ at $4^{\circ} \mathrm{C}$, and stained using 1:20 diluted FITC-labeled mouse anti-mouse secondary antibody for $15 \mathrm{~min}$ at $4^{\circ} \mathrm{C}$. The fluorescence intensity was analyzed with Cellquest software (Becton Dickinson Bioscience, Franklin Lakes, NJ, USA). The cells that were incubated with only secondary antibody were analyzed as the controls.

Cell proliferation assay. For the proliferation assays, Cell Titer 96 AQ Non-Radioactive Cell Proliferation Assay kits (Promega, Madison, WI, USA; no. G5421) were used. SK-LMS-1, NIH 3T3, CNE-1, CNE-2, HK-1, HONE-1 and SUNE-1 cells were plated in 96-well plates with 500 cells per well in triplicate in serum-free medium for $12 \mathrm{~h}$. Three doses of HGF $(0.01$, 0.1 and $1 \mu \mathrm{g} / \mathrm{ml}$ ) were added $12 \mathrm{~h}$ later while adhered to the bottom of the wells. The cells were incubated with HGF for $24 \mathrm{~h}$ prior to the addition of the MTS reagent. The plate was then read using a Dynex spectrophotometer. Each experiment was repeated three times and the average and standard error of the mean (SEM) were calculated.

Scratch wound closure assay. After being maintained in quiescence in serum-free culture medium for $24 \mathrm{~h}$, confluent cell monolayers of HONE-1 in 6-well plates were wounded by mechanical scraping with a $200 \mu 1$ pipette tip. Following washing to remove cell debris, cultures were incubated in RPMI-1640 with or without HGF at a concentration of $0.1 \mu \mathrm{g} / \mathrm{ml}$. Wound width was assessed at the time of scraping to ensure that all wounds were the same at the start of the 

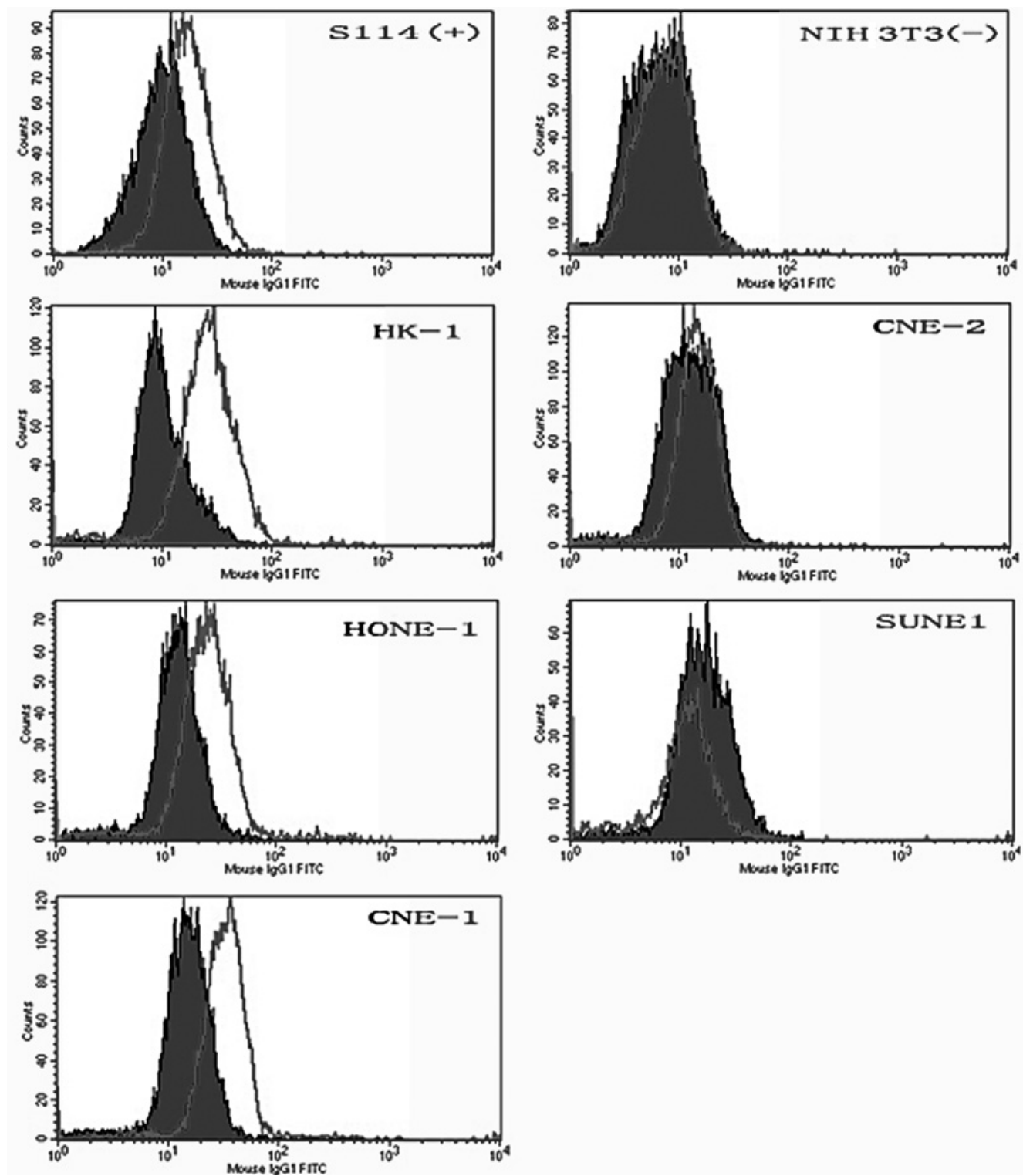

Figure 3. Anti-Met25 specifically bound to the Met-expressing cells, HONE-1, HK-1 and CNE-1, which is demonstrated by the shifts in the FACS images. There was a greater shift in the S114 cells compared to the other cell lines, used as positive controls. However, there was no shift in the NIH 3 T3 cells, used as negative controls. FACS, fluorescence-activated cell sorting.

experiment. The wound closure was recorded photographically over time, using phase-contrast microscopy 24 and 48 h later.

Statistical analysis. The assays were performed in triplicate. Data were expressed as the mean \pm SD. Statistical analyses were performed using analysis of variance by SPSS 14.0 software. $\mathrm{P}<0.05$ was considered to indicate a statistically significant difference.

\section{Results}

HGF gene expression in different NPC cells. RT-PCR was used to determine HGF gene expression in several NPC cell lines (CNE-1, CNE-2, HK-1, HONE-1 and SUNE-1). There was no HGF gene expression in any of the NPC cell lines, with the exception of the S114 cells, which served as the positive controls (Fig. 1). The results suggest that further study is required to determine the expression of the HGF ligand to investigate the role of HGF in NPC cell lines.

Met 25 protein expression in different NPC cells. Since the HGF gene was not expressed in the NPC cells, western blot analysis was used to detect the expression of the HGF ligand, Met25 protein. A comparison of the S114 cells (positive control) and NIH 3T3 cells (negative control) resulted in the detection of Met25 protein expression in the HONE-1, HK-1 and CNE-1 cells, but not in the CNE-2 and SUNE-1 cells (Fig. 2).

Anti-Met25 binding activity to the cell surface. The specific binding activity of anti-Met25 mAb to the Met protein on viable Met-expressing cells was determined by FACS analysis. FITC-conjugated anti-Met $25 \mathrm{mAb}$ specifically bound Met-expressing S114, HONE-1, HK-1 and CNE-1 cells (Fig. 3). No binding was observed in the NIH $3 \mathrm{~T} 3$ cells, which were the 


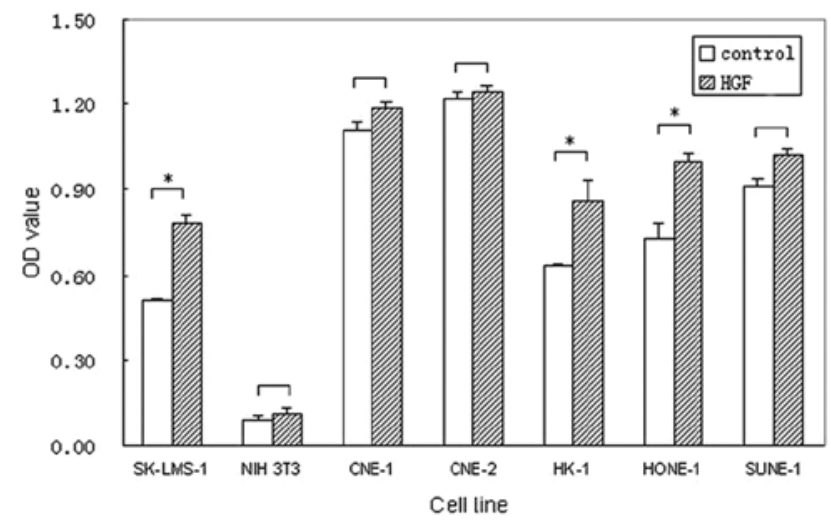

Figure 4. Tumor cell proliferation following HGF stimulation. CNE-1, CNE-2, HK-1, HONE-1 and SUNE-1 tumor-derived cells were plated in 96-well plates in serum-free medium for $12 \mathrm{~h}$. HGF was added to the cells at different doses and plates were incubated for $24 \mathrm{~h}$. MTT reagents were added and the absorbance was read at $492 \mathrm{~nm}$. There was a significant increase in the HONE-1 and HK-1 cells when treated with HGF $(\mathrm{P}<0.05)$. HGF, hepatocyte growth factor; MTT, 3-(4,5-dimethylthiazol-2-yl)-2,5-diphenyltetrazolium bromide; OD, optical density.

negative controls. Similar results were obtained from the two parts of the experiment by western blot and FACS analyses.

HGF promotes the proliferation of NPC cells. To investigate the role of HGF in NPC cells, HGF was used as an exogenous factor to determine whether it was capable of stimulating cell proliferation. Three different concentrations $(0.01,0.1$ and $1 \mu \mathrm{g} / \mathrm{ml}$ ) of HGF were used. Concentrations of 0.1 and $1 \mu \mathrm{g} / \mathrm{ml}$ HGF promoted the proliferation of NPC cells, but not the CNE-1, CNE-2 and SUNE-1 cells. The concentration of $1 \mu \mathrm{g} / \mathrm{ml}$ HGF had similar effects on the cells compared with $0.1 \mu \mathrm{g} / \mathrm{ml}$. However, $0.01 \mu \mathrm{g} / \mathrm{ml}$ HGF did not markedly stimulate the proliferation of all NPC cells. As shown in Fig. 4, exogenous HGF $(0.1 \mu \mathrm{g} / \mathrm{ml})$ promoted the proliferation in the NPC cell lines with the exception of CNE-1, CNE-2 and
SUNE-1, with significant effects in HONE-1 and HK-1 (36.5 and $35.5 \%$ ) (data of 1 and $0.01 \mu \mathrm{g} / \mathrm{ml}$ HGF were not shown).

HGF promotes the healing of scrapewound. The administration of HGF, a specific Met ligand, significantly promoted wound closure in the experimental NPC cell lines and had a profound effect on migration, which plays a pivotal role in wound healing processes. Activation of Met increased migration in in vitro scrape-wounding assays in HONE-1 cells (Fig. 5).

\section{Discussion}

NPC is the most commonly diagnosed malignancy in Southern China (1). The combination of radiotherapy and chemotherapy is not always effective for early resistant NPC and locoregional advanced cases, therefore other treatment choices are required to prevent treatment failure, including cases of distant metastasis. Targeted treatment has become a new way to treat NPC due to its availability and safety. The approach of adding epidermal growth factor receptor (EGFR)-targeted therapy to radiotherapy and chemotherapy is being actively studied for locoregionally advanced NPC, on the basis of its overexpression and poor survival outcome (11).

The aim of this study was to determine the role of HGF in several NPC cell lines, and to obtain evidence to improve prognosis results and decrease sequela as radiation alone cannot kill hypoxic cancer cells. The HGF/c-Met signal pathway is related to the above, as hypoxia was able to activate this pathway and result in migration and metastasis (12). HGF can lead to the phosphorylation of two tyrosine residues at the c-terminus of the Met protein upon binding to Met, which subsequently promotes cancer growth and metastasis $(13,14)$. Met phosphorylation also induces the tyrosine phosphorylation of $\beta$-catenin, which causes $\beta$-catenin dissociation from E-cadherin (15). HGF may contribute to cell invasion by modulating E-cadherin-mediated cell-cell adhesion through the downregulation and internalization of E-cadherin.

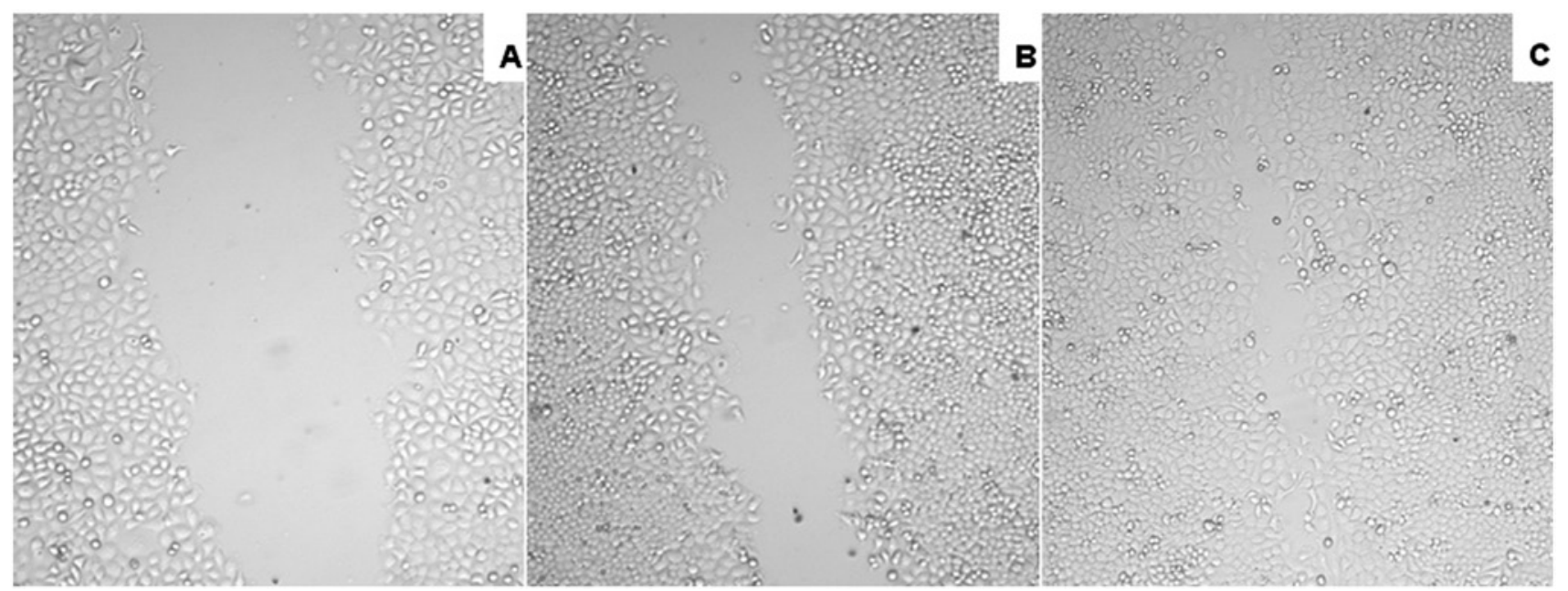

Figure 5. HGF significantly promoted wound closure in the experimental NPC cell lines and had a profound effect on the migration. Met increased migration in scrape-wound assays performed on HONE-1 cells. (A) Without HGF; (B) incubated with HGF ( $0.1 \mu \mathrm{g} / \mathrm{ml})$ for $24 \mathrm{~h}$; (C) incubated with HGF (0.1 $\mu \mathrm{g} / \mathrm{ml})$ for $48 \mathrm{~h}$. HGF, hepatocyte growth factor; NPC, nasopharyngeal carcinoma. 
The results of this study have demonstrated that the selected NPC cells did not express the HGF gene, with S114 cells serving as the positive control cell line. To investigate the role of HGF in NPC cell lines, we detected the expression of the HGF ligand, Met25 protein in the above cells. The results have shown that only the HK-1, HONE-1 and CNE-1 cells expressed the Met protein, whereas the CNE-2 and SUNE-1 cells did not express this protein. These results were validated by similar results obtained from the FACS analysis where only the HK-1, HONE-1 and CNE- 1 cell lines bound the anti-Met $25 \mathrm{mAb}$, whereas the CNE-2 and SUNE-1 cells did not exhibit binding activity. These results suggest that, unlike the CNE-2 and SUNE-1 cells, the HK-1, HONE-1 and CNE-1 cells may express segments of the Met protein, . This observation indicates that HGF affects the cells that express the Met protein. These results provide evidence that HGF may be selected as an exogenous factor to investigate its role in promoting the proliferation of NPC cells.

A study by Aune et al revealed that patients with ovarian carcinomas had higher serum HGF levels than patients with borderline and benign ovarian tumors (16). HGF in serum is an indicator of ovarian carcinoma in females with a pelvic mass, and with poor prognosis in advanced ovarian cancer. Similar results have been demonstrated in studies evaluating HGF in gastric carcinoma, colorectal cancer and hepatocellular carcinoma (17-19). However, there has been no study evaluating HGF in NPC. In this study, we aimed to determine whether the Met receptor was expressed in several types of NPC cells despite no expression of the HGF gene. Results suggested that the Met receptor was activated by its paracrine ligand, HGF, from the interstitial tissues rather than by an autocrine loop or its activating mutation.

To determine the functions of the HGF/c-Met pathway in NPC cells, the effects of exogenous HGF were observed. Results from proliferation assays revealed that HGF promoted the proliferation of HK-1 and HONE-1 NPC cells. Additionally, exogenous HGF was found to significantly increase the proliferation of the HONE-1 and HK-1 cells (36.5 and 35.5\%, respectively, $\mathrm{P}<0.05$ ). To validate the effect of HGF, healing of scrape-wounds on HONE-1 cells was determined. As over 95\% of biopsies are classified as WHO type II or III, which are poorly differentiated, the HONE-1 cell line was selected for the migration experiments. HGF was found to significantly promote wound closure in HONE-1 cells and had a profound effect on the migration. The results indicate that Met may be a good target for treatment, as it is capable of decreasing the proliferation and migration of NPC cells.

In conclusion, our study has shown that exogenous HGF promoted the proliferation of NPC cells, and that c-Met may serve as a ligand for HGF and be an effective target for blocking the function of HGF. This finding may therefore lead to the development of new drugs for NPC treatment. Further investigations are required to understand the mechanisms of the HGF/c-Met pathway in NPC.

\section{Acknowledgements}

The authors thank Rich West for running the fluorescenceactivated cell sorting Calibur cytometer. This study was supported by the National Natural Science Foundation of China (81101671 and 30570785).

\section{References}

1. Spano JP, Busson P, Atlan D, Bourhis J, Pignon JP, Esteban C and Armand JP: Nasopharyngeal carcinoma: an update. Eur J Cancer 39: 2121-2135, 2003.

2. Lai SZ, Li WF, Chen L, et al: How does intensity-modulated radiotherapy versus conventional two-dimensional radiotherapy influence the treatment results in nasopharyngeal carcinoma patients? Int J Radiat Oncol Biol Phys 80: 661-668, 2011.

3. Lee AW, Ng WT, Hung WM, et al: Major late toxicities after conformal radiotherapy for nasopharyngeal carcinoma-patientand treatment-related risk factors. Int J Radiat Oncol Biol Phys 73: 1121-1128, 2009.

4. Chan AT, Leung SF, Ngan RK, et al: Overall survival after concurrent cisplatin-radiotherapy compared with radiotherapy alone in locoregionally advanced nasopharyngeal carcinoma. J Natl Cancer Inst 97: 536-539, 2005.

5. Wei KR, Xu Y, Liu J, Zhang WJ and Liang ZH: Histopathological classification of nasopharyngeal carcinoma. Asian Pac J Cancer Prev 12: 1141-1147, 2011.

6. Trusolino L and Comoglio PM: Scatter-factor and semaphoring receptors: cell signalling for invasive growth. Nat Rev Cancer 2: 289-300, 2002.

7. Qian CN, Guo X, Cao B, et al: Met protein expression level correlates with survival in patients with late-stage nasopharyngeal carcinoma. Cancer Res 62: 589-596, 2002.

8. Rong S, Oskarsson M, Faletto D, et al: Tumorigenesis induced by coexpression of human hepatocyte growth factor and the human Met protooncogene leads to high levels of expression of the ligand and receptor. Cell Growth Differ 4: 563-569, 1993.

9. Jeffers M, Rong S and Vande Woude GF: Enhanced tumorigenicity and invasion-metastasis by hepatocyte growth factor/ scatter factor-met signaling in human cells concomitant with induction of the urokinase proteolysis network. Mol Cell Biol 16: 1115-1125, 1996.

10. Cao B, Su Y, Oskarsson M, et al: Neutralizing monoclonal antibodies to hepatocyte growth factor/scatter factor (HGF/SF) display antitumor activity in animal models. Proc Natl Acad Sci USA 98: 7443-7448, 2001.

11. Chua DT, Wei WI, Wong MP, Sham JS, Nicholls J and Au GK: Phase II study of gefitinib for the treatment of recurrent and metastatic nasopharyngeal carcinoma. Head Neck 30: 863-867, 2008.

12. Eder JP, Vande Woude GF, Boerner SA and LoRusso PM: Novel therapeutic inhibitors of the c-Met signaling pathway in cancer. Clin Cancer Res 15: 2207-2214, 2009.

13. Arriola E, Cañadas I, Arumí-Uría M, et al: MET phosphorylation predicts poor outcome in small cell lung carcinoma and its inhibition blocks HGF-induced effects in MET mutant cell lines. Br J Cancer 105: 814-823, 2011.

14. Syed ZA, Yin W, Hughes K, Gill JN, Shi R and Clifford JL: $\mathrm{HGF} / \mathrm{c}-\mathrm{met} / \mathrm{Stat} 3$ signaling during skin tumor cell invasion: indications for a positive feedback loop. BMC Cancer 11: 180, 2011.

15. Xie LQ, Bian LJ, Li Z, Li Y, Li ZX and Li B: Altered expression of E-cadherin by hepatocyte growth factor and effect on the prognosis of nasopharyngeal carcinoma. Ann Surg Oncol 17: 1927-1936, 2010.

16. Aune G, Lian AM, Tingulstad S, Torp SH, Forsmo S, Reseland JE and Stunes AK: Increased circulating hepatocyte growth factor (HGF): a marker of epithelial ovarian cancer and an indicator of poor prognosis. Gynecol Oncol 121: 402-406, 2011.

17. Tanaka K, Miki C, Wakuda R, Kobayashi M, Tonouchi H and Kusunoki M: Circulating level of hepatocyte growth factor as a useful tumor marker in patients with early-stage gastric carcinoma. Scand J Gastroenterol 39: 754-760, 2004.

18. Toiyama Y, Miki C, Inoue Y, Okugawa Y, Tanaka K and Kusunoki M: Serum hepatocyte growth factor as a prognostic marker for stage II or III colorectal cancer patients. Int J Cancer 125: 1657-1662, 2009.

19. Mizuguchi T, Nagayama M, Meguro M, et al: Prognostic impact of surgical complications and preoperative serum hepatocyte growth factor in hepatocellular carcinoma patients after initial hepatectomy. J Gastrointest Surg 13: 325-333, 2009. 